\title{
Mind the gap! - Über Wissen und Nichtwissen im Design
}

\author{
Oder: Es gibt nichts Theoretischeres als eine gute Praxis
}

\author{
Wolfgang Jonas
}

Die Frage nach den Grundlagen oder dem „,common ground“ des Designs ist weiter offen. Grundlagen sind entweder „Nichts“ - der Beginn der kulturellen Evolution, der „Nullpunkt“ (der Stock und der Stein des Primaten) - oder „Alles“ - die Geschichte dessen, was bis heute passiert ist. Vom „Nullpunkt“ bis heute hatten wir einen endlosen Zyklus/eine Spirale/eine „Geschichte“ der Konstruktion und Dekonstruktion von Artefakten und Wissen, oder: der Komplexifikation (um das überladene Wort „Lernen“ vorerst zu vermeiden). Und wir haben die fortschreitende ,Wellenfront“ der Gegenwart, den Ort, an dem wir die Ähnlichkeit von Entwerfen und „science in action“ erfahren können. Beide Prozesse agieren im hybriden Sumpf der Artefakte, Bewusstseine, Kommunikationen und menschlichen Körper. Bedeutung, Status und Gebrauch von Wissen sind jedoch im Design und in den Wissenschaften grundsätzlich verschieden. Wissenschaft zielt auf Vorhersagbarkeit und braucht deshalb stabile Modelle, die „das Gleiche“ liefern. Wissenschaft muss ihre Modelle „reinigen“, um sie aus Hypothesen in Vorhersagemaschinen zu verwandeln. Körper, Bewusstseine, Kommunikationen und Artefakte werden säuberlich voneinander getrennt. Wissenschaftliche Probleme sind gelöst, solange die Lösung sich nicht als falsch herausstellt, d.h. als weniger erklärungskräftig im Vergleich zu einer neuen Lösung. Design dagegen zielt auf Einzelphänomene, welche zu vielfältigen, unvorhersehbaren Bedingungen passen sollen. Design muss bewusst Variationen herstellen, Differenzen schaffen, weil die „Passungen“ sich auflösen, verschwinden, altmodisch werden. Designumgebungen verändern sich zu schnell, um sinnvoll von wahrem oder falschem Wissen zu sprechen. Das Archiv von Designwissen ist wie ein Gedächtnis, ein wachsendes Reservoir von ermöglichenden wie beschränkenden Erfahrungen. Expertise im Design besteht in der Kunst des gleichzeitigen Umgangs mit wissenschaftlichem und nichtwissenschaftlichem Wissen, mit unscharfem und veraltetem Wissen und mit Nichtwissen, um diese wertgeladenen Passungen zu realisieren. Mit anderen Worten: in der „Kunst des Durchwurstelns“.

Wir stehen vor der paradoxen Situation der wachsenden Manipulationsmacht durch Wissenschaft und Technologie bei gleichzeitig abnehmender prognostischer Kontrolle über die sozialen Konsequenzen. Die Grenzen dieser projektorientierten Wissenschaft deuten auf eine Anpassung der Rolle des Designs hin: bescheidener und anmaßender zugleich. 
Meine konzeptionellen Werkzeuge zum Verstehen der Mechanismen der Produktion und Zerstörung von Artefakten und Wissen umfassen: (1) soziologische Systemtheorie (Luhmann) mit dem Konzept der Autopoiesis, einschließlich des Wechsels von Identitäts- zu Differenzdenken, (2) Evolutionstheorien (Darwin, Luhmann), (3) evolutionäre Erkenntnistheorie (Campbell, Riedl) sowie das Konzept der Aktionsforschung.

\section{$1 \quad$ Warum immer noch Designgrundlagen?}

Der Diskurs über Designgrundlagen ist isoliert und erratisch. Die geschäftigen und atemlosen „wissenschaftlichen“ Designforschungsaktivitäten müssen durch einen kohärenteren und flexibleren und weniger rigiden theoretischen Rahmen gestützt werden, um die Autonomie und den spezifischen Charakter von Design (-forschung) zu befördern und zu klären.

Die Frage nach den Grundlagen habe ich mehrfach behandelt (Jonas, 1999, 2000). Der erste Artikel beschreibt Designtheorie, ähnlich wie Design selbst, als floating network of chunks of ideas, ohne festen epistemologischen Kern, agierend in der Interfaceregion zwischen sich ständig verschiebenden Bezugssphären: dem Kontextuellen und dem Artefaktischen. Einige provokante Konsequenzen wurden herausgestellt, etwa: es gibt keinen Fortschritt im Design, oder: Design ist amoralisch und muss es sein, um seine Funktion erfüllen zu können. Der zweite Artikel beschreibt das genannte Interface in Anlehnung an Schön (1983) als „Sumpf“, eine provisorische Metapher für den hybriden Mix aus Natürlichem, Menschlichem und Göttlichem (Latour, 1998), der keine Grundlagen bieten kann, sondern nur Einstiegspunkte. Design hat keine Grundlagen, weil das Entwerfen selbst die grundlegende menschliche Aktivität darstellt. Grundlagen können als emergente kurzlebige Muster hervortreten. Wissenschaft agiert ebenfalls in diesem Sumpf (der dort Labor oder Feld genannt wird), aber sie ist gezwungen, die dort produzierten Fakten zu „reinigen“ und zu de-kontextualisieren, um ihr mythisches Bild zu bewahren, dichter an der Wahrheit zu sein als andere Arten der Wissenserzeugung. Und ich habe gefragt, ob Design diesem verlockenden, aber problematischen Programm der Wissenschaftlichkeit folgen sollte.

Design hat zweifellos beachtlichen Status und hohe Komplexität erreicht:

- Dutzende von Professionen benutzen das Konzept Design. Aber worin bestehen ihre Gemeinsamkeiten, abgesehen von der Überführung existierender in neue (bestenfalls gewünschte) Situationen?

- Es gibt einen wachsenden Bereich der Designforschung. Aber worin besteht das designspezifische dieser Aktivitäten, abgesehen davon, dass sie für Designaufgaben hilfreich sein mögen?

- Es werden enorme, fast „kanonische“ Listen von designrelevanten Wissensbeständen und Disziplinen, verbunden mit wohl etablierten akademischen Regeln propagiert (Friedman, 2001). Aber schafft dies tatsächlich „common ground“ oder verstellt es nicht eher durch Quantität und Eloquenz den Blick auf die fehlenden eigenen Grundlagen und Regeln? 
Ich will nicht als Spielverderber auftreten und auch nicht die gegenwärtigen Anstrengungen und Erfolge der Designforschung in all ihren Facetten in Frage stellen, aber angesichts des Dargestellten erscheint das laute Propagieren von gemeinsamem Grund und von Fortschritt im Design wie eine Beschwörungsformel. Es mag sinnlos erscheinen oder gar engstirnig, auf diesem Punkt zu beharren, aber ich halte es für notwendig, die Frage nach den Grundlagen offen und lebendig zu halten, um eine spezifische Designidentität entwickeln zu können. Diese umfasst ontologische, epistemologische und methodologische Aspekte:

1. Gibt es ein Wesen von Design/Entwerfen?

2. Was ist die allgemeine Funktion von Design?

3. Worin besteht die spezifische Natur von Wissen im Design?

4. Wie steht es mit der Beziehung Design/Wissenschaft?

5. Wie lässt sich der Prozess der „Problemlösung“ durch Forschung verbessern?

Das angeblich zentrale Produkt des Entwerfens, das Artefakt, fehlt hier. In meiner Sicht sind Artefakte kontingente Materialisierungen in dem endlosen Prozess, welche bestenfalls retrospektiv (zweifellos mit Nutzen für weitere Projekte) interpretiert werden können.

Nach der „Common Ground“-Konferenz der Design Research Society im September 2002 gab es eine kurze Diskussion über die kritische Äußerung, dass für einige Leute die Konferenz nicht den durch die ambitionierte Metapher (common ground) geweckten Erwartungen entsprochen habe. Die Debatte wurde durch die lakonische Antwort der Organisatoren abgebrochen, es habe sich um ,a title, not a theme“ gehandelt. Dies ist höchst unbefriedigend, wenn man bedenkt, dass theoretische Beiträge, welche explizit die Frage der Grundlagen ansprechen, einen regelrechten „Karneval der Meinungen“ in diesem Feld offenbaren (Jonas, 2001-2003, 2004). Wir sollten die Fallen und Sackgassen dieses Unternehmens im Auge behalten. Die Rede von „common ground“ impliziert den Anspruch, das Ganze des Designs von einer Position innerhalb des Designs zu beschreiben. Normative Fixierungen und quasireligiöse Formeln sind häufige Folgen derartiger autologischer Situationen von Selbstreferenz und Paradoxie. Sie verdecken die notwendige Einzigartigkeit und Fluidität aller Selbstbeobachtungen und verleiten uns dazu, unsere Landkarte für die Landschaft zu halten.

Kann es Grundlagen mit Axiomen, Gesetzen und wissenschaftlichen Methoden geben? Oder müssen wir die Methode des eleganten „muddling through“ akzeptieren? Jeder Versuch der Fixierung von Grundlagen führt zur Frage: was ist die Grundlage dieser Grundlagen? Das „Münchhausen-Trilemma“ taucht auf (Albert, 1968) und bietet die Auswege des infiniten Regresses, der Zirkularität oder der dogmatischen Setzung. Love (2002) sucht auf der neurophysiologischen Ebene nach Grundlagen. Seine biologisch basierten Erklärungen werden im infiniten Regress enden, so ist zu befürchten. Buchanan (2001) führt „generative principles“ ein, doch woher kommen diese? Sie sind selbstverständlich generiert, aber sie offenbaren einen reizvollen zirkulären Aspekt, wie gezeigt werden wird. Friedman (2001) definiert einige Begriffe und folgert: es gibt Grundlagen und basta! Meiner Ansicht nach scheint eine Mischung aus Zirkularität + Zeit (,,Spiralität“) am meisten zu versprechen. 


\section{$2 \quad$ Grundlagen durch Definition und Deduktion?}

Wir neigen zu der unangemessenen Erwartung, polymorphe Phänomene (Design, Fortschritt, Grundlagen) mittels geschärfter Definitionen und formaler Schlussfolgerungen in den Griff zu bekommen. Unscharfe Konzepte sind jedoch möglicherweise besser greifbar, indem der Kern des Phänomens mit Hilfe von Analogien illustriert wird. Dies bedeutet die Verschiebung von einer definitorischen (binären) in Richtung auf eine transitorische (fuzzy) Logik.

Friedman (2001) hat die erwähnten Artikel (Jonas 1999, 2000) kritisch gelesen. Das Folgende ist Teil eines imaginären Dialogs (Jonas, 2002), d.h. ich antworte auf seine Kritik und konzentriere mich auf die Frage des allgemeinen Stils und die Fragen nach Fortschritt und Grundlagen. Die hauptsächliche Kritik lautete: „The arguments against the concept of foundations are intuitionist in nature. “

K.F.:

„, [...] These papers outline problems and issues without defining them. Opening the problem space allows us to reflect. Closing the problem space through robust definitions allows us to begin the search for solutions."

W.J.:

The basic problems mentioned cannot be defined in a manner you would accept; definitions are not available. The concept of interface clearly refers to Simon and Alexander and their notions of design as interface discipline. „Robust“ definitions might kill the problem before the search for solutions has even started. The idea that the problem space has to be closed in order to proceed towards solutions is inappropriate. Since the early 1970 s we could know that in ill-defined, wicked problem situations problems and solutions evolve in a parallel process. If at all, the problem can be stated when a solution is achieved. And then the solution is the problem! I am convinced that this is true for design problems as well as for design theory problems.

\section{K.F.:}

„The growth of design knowledge, the steady history of improvements in design practice, the dramatic development of design research, and the gradual development of design teaching, all indicate progress.

Progress is not uniform. Comprehensive progress is impossible. Nevertheless, there is relatively wide agreement in our field that we are meeting Bunge's (1999, S. 227) definition of progress as a 'process of improvement in some regard and to some degree" in all four areas of design.

The state of physics in 1895 offers a good comparison for our field. Because we are a different kind of field, we cannot hope to make the fundamental progress that physics has made over the past 100 years. Even so, we can hope to grow if we focus on a progressive research program. 


\section{$[\ldots]$}

Progress in research and in practice depends on prior art. This is another way of stating that progress requires foundations. If there is progress - and there is - there must be foundation(s). There is progress in design. QED: design has foundations.“

\section{W.J.:}

There is an interesting rule, or axiom: „Progress requires foundations.“ And as we have progress, we have foundations. This is a nice circle, or syllogism/deduction, which fails immediately if one does not believe in your definition of progress and your postulation of progress in design. In my old-fashioned view progress comprises (1) an increase in scientific „truth" (there is progress e.g. in physics; but in design?), (2) an improvement of the human condition, the claim that Galilei and Bacon stated for science (there is progress in many fields; but through design?), and, (3) the utopian claim of enlightenment thinking: better human beings (no progress here!). But I do not accuse design for not showing much progress in this sense, because, as I argued, design is the agency of bridging the gap, the interface. There is no reference point for defining progress, but merely fit or non-fit. Is Mac OS X a design progress compared with OS 9, or just an increase in functional complexity?

Parallels with physics or even mathematics seem inappropriate. Maybe there are parallels to the situation of the Design Methods Movement (and the „design science decade“) some 40 years ago: an exponential growth in rigidity [...] and then a collapse with important insights: that there are designerly ways of knowing, that design problems are mostly ill-defined, embedded, situated, etc.

Heute sind wir in einer Situation, wo andere Disziplinen den fragilen, fluiden, geschichtlichen Charakter ihrer jeweiligen „Grundlagen“ erkennen. Wir sollten uns vor der „fallacy of misplaced concreteness“ (A.N. Whitehead) hüten, also vor der Annahme, wenn wir nur ein Wort besitzen, dann gäbe es folglich auch ein Ding, welches von diesem Wort bezeichnet wird. Um es einfach auszudrücken: Worüber reden wir eigentlich, wenn wir über Design und Designforschung reden?

Ich lehne „systematische“ Forschung keinesfalls ab. Aber dies muss nicht notwendigerweise dasselbe bedeuten wie etwa in den Sozialwissenschaften. Erfrischende und inspirierte designerische Forschungsweisen sind durchaus möglich (Dunne \& Raby, 2001). 


\title{
3 Grundlagen durch „Generative Prinzipien“?
}

\author{
„Generative Prinzipien“ sind zuallererst generierte Prinzipien. Um den Grundlagen \\ näher zu kommen, müssen wir die darunter liegenden evolutionären Mechanismen \\ anschauen, die in der Lage sind „Prinzipien zu generieren“.
}

Buchanan's (2001) ,ecology of culture“ könnte gut mit meinem berüchtigten „Sumpf“ verglichen werden. Wenn er sagt, dass „We tend to dismiss the way human beings have formed their beliefs in response to the natural and human environment", dann führt er explizit ein evolutionäres Konzept ein. In der Entwicklung unserer Denkwege und -weisen sind wir von den philosophischen Annahmen abhängig, die hinter unseren grundlegenden Überzeugungen stehen. Deren Kontingenz wird jedoch niemals explizit gemacht; sie bleiben meist in einem vorbewussten Zustand. Um sie deutlicher zu machen, identifiziert oder erfindet Buchanan vier ,generative Prinzipien“ als Erzeuger für die vielfältigen, manchmal inkompatiblen Muster des gegenwärtigen Theoretisierens im Design. Sein Schema zeigt zwei Dimensionen: die phänomenalen Prozesse (A) und die ontischen Bedingungen (B), jeweils mit zwei typischen Ausprägungen, so dass ein ordentliches Kreuzschema entsteht, ein Beispiel für Theorie als Design:

\section{A: Phänomenal.}

Die zugrunde liegende Annahme ist, ,that design is best understood by our experience of it [...].“

A1: Erfahrung und Umwelt.

Der Schwerpunkt liegt ,on the problems that human beings encounter in their environment. [...] It seeks to identify and integrate multiple causes of design rather than reducing it to a single cause. [...] “Die vier Aristotelischen causae zeigen sich hier.

\section{A2: Agent.}

Der Schwerpunkt liegt auf ,the agent who performs an action. Design is shaped by the actions that human beings take in creating and projecting meaning into the world. [...] This existential, operational approach is exemplary in its key features. It looks for successful examples of design practice in the past or present for models that may guide future ventures in designing. [...]“

B: Ontisch.

Die zugrunde liegende Annahme lautet, es gebe ,'real and ultimate conditions that determine design in human experience $[. .$.$] “"$

B1: Zugrunde liegende Kräfte.

Der Schwerpunkt liegt auf ,underlying natural forces and material reality. [...] The paradigm of design is engineering, since engineering is closest to the natural conditions that are the 'real and ultimate" conditions of human life. [...] This [...] approach [...] looks to the conditions that have shaped the past and seeks to project the trends of fundamental forces and movements into the future $[\ldots]$ “. 


\section{B2: Transzendente Ideen.}

Hier liegt der Fokus auf ,ideas and ideals that transcend the necessities and contingencies of physical or material culture and the limitations of individual, personal experience. [...] This vision [...] is always oriented toward an ideal of beauty, truth, or justice that transcends and permeates the world of human experience, giving structure to meaning and values. [...]“

Auf diese Weise wird eine klärende Struktur für das chaotische Bild entworfen, welches Designtheoriebildung heute bietet. Das Schema als Ganzes hat eine stark Platonische Anmutung, was Buchanan nur dem Prinzip B2 zuschreibt. Es scheint in einem ewigen Reich der Ideen zu schweben und dabei die verwirrende Vielfalt der phänomenalen Welt der Designtheorien zu produzieren. Aber woher kommt es selbst? Kann es vielleicht in ein generativeres Modell der Wissensproduktion integriert werden? Die Antwort ist in dem Schema selbst enthalten. Buchanan - zwischen den Zeilen wird dies deutlich - scheint das Prinzip A1: Erfahrung und Umwelt zu favorisieren. Menschliche Erfahrungen lassen persönliche Verhaltensweisen, Präferenzen, Stile entstehen. In der Folge werden sich, entsprechend diesen Präferenzen, Theorien darüber, wie die Welt (oder Design) funktioniert, herausbilden. Buchanan's vier Prinzipien sind eine dieser emergenten Theorien, welche im Gegenzug durch ihre Verbreitung (Design Issues ist ein effizienter Replikator) persönliche Verhaltensweisen, Präferenzen und Stile in der Community und damit die weiteren Bedingungen unserer Erfahrungen beeinflussen. „Generative Prinzipien“ sind generiert, auf dem evolutionären Hintergrund von 2500 Jahren westlicher Philosophie.

Um dies zusammenzufassen: Das generative Prinzip A1 scheint ein wenig „grundlegender“ zu sein als die anderen, denn es enthält sowohl die anderen vier wie auch sich selbst bereits. Dies zeigt den fraktalen Charakter und die Selbstreferenz der Designtheorie und - dies ist wichtig - erlaubt es uns, dieses „Buchanan Mem“ in den allgemeinen Prozess der Wissensgenerierung zu integrieren.

\section{Eine Grundlage: Evolutionäre Erkenntnistheorie}

Die evolutionäre Erkenntnistheorie liefert den elementarsten generativen Mechanismus zum Verständnis des Lernens in der lebenden Welt und erklärt auf diese Weise die fortlaufende Produktion und Reproduktion von Artefakten und von Wissen, schließlich von Design und Wissenschaft als dynamische Formen.

Dies ist das „,Wesen“ ( $\rightarrow$ Frage 1).

Es besteht keine Notwendigkeit für irgendeine spezifische Natur von Wissen im Design $(\rightarrow$ Frage 3). 
Ein Darwinscher Mechanismus von (1) Mutation - (2) Selektion - (3) Re-Stabilisierung - und so fort wird sichtbar: (1) Jonas führt ein neues Konzept ein, das als Mutation, kreativer Akt, beabsichtigte Provokation bezeichnet werden kann, je nachdem, was man als Beobachter bevorzugt. (2) Friedman agiert als selektive Umgebung, indem er die Aussage in Frage stellt. (3) Der Ideenklumpen übersteht diesen „Überlebenskampf“, die Interaktion zwischen dem System (Jonas' Ideen) und dem Kontext (Friedmans Kritik). Das Konzept ist re-stabilisiert. (1') Buchanans neuer attraktiver Ideenklumpen erscheint und Jonas versucht, ihn zu integrieren. (2') Jemand könnte nun wiederum als selektiver Mechanismus agieren und so fort. Im Unterschied zu einem genetischen handelt es sich hier um einen memetischen Prozess. Die „Ideenklumpen“, welche übertragen/fortentwickelt werden, können als Meme oder Memplexe (Dawkins, 1976; Blackmore, 1999) angesehen werden. Die wesentliche Beobachtung bis hierher ist, dass sich in Designdebatten Lernzyklen (ich wechsle zum geläufigeren „Lernen“) entwickeln könnten, wenn es nur Debatten dieser Art gäbe.

Die Basis unserer Lernprozesse, welche aus meiner Sicht den epistemologischen Kern des Designs darstellen, kann als biologisch betrachtet werden, verankert in der Notwendigkeit von Organismen, in einer Umgebung zu überleben. Das Ziel kann nicht in der wahren Repräsentation einer externen Realität bestehen, sondern in ihrer (Re-) Konstruktion zum Zweck der angemessenen (Re-) Aktion. Schon Aristoteles vermutete, dass die Erkennbarkeit der Welt darauf beruhen müsse, dass es eine Art von Ähnlichkeit zwischen den „Partikeln“ der Welt und denjenigen in unseren Sinnen gibt. Die Geschichte der biologischen Evolution legt tatsächlich Übereinstimmungen zwischen der Struktur der materiellen Welt und der Art und Weise nahe, wie wir diese wahrnehmen und über sie denken. Evolutionäre Erkenntnistheoretiker (Campbell, 1974) behaupten, dass das Kantische transzendentale Apriori durch die Annahme einer evolutionären Passung zwischen den Objekten und den Subjekten des Erkennens ersetzt werden müsse.

Das evolutionäre Modell der Wissensproduktion zeigt die Form eines Spiralschemas mit struktureller Identität von der molekularen bis zur kognitiven und kulturellen Ebene (Riedl, 2000). Die Grundstruktur besteht in einem Kreis aus Versuch (Erwartung) und Erfahrung (Erfolg oder Fehlschlag, Bestätigung oder Widerlegung), von Aktion und Reflektion. Ausgehend von vergangenen und getrieben von neuen Fällen besteht der Zirkel aus einem induktiven/heuristischen Halbkreis mit zielgerichtetem Lernen aus der Erfahrung, hinführend zu Hypothesen und Theorien und Prognosen darüber, wie die Welt funktioniert, und einem deduktiven/logischen Halbkreis mit der Bestätigung oder Widerlegung der Theorien. Je nach den Zwecken und erwünschten Ergebnissen haben wir es mit Design oder mit Wissenschaft zu tun.

Erst sehr spät im Laufe der kulturellen Evolution wurde dieses allgemeine Schema aufgespaltet in die ratiomorphen (ein von Konrad Lorenz geprägter Begriff) Systeme des Erkennens einerseits und die rationalen Systeme des Erklärens/Verstehens andererseits, mit dem Extrem im logischen Positivismus: dem Dualismus von „Entdeckungszusammenhang“ (Handeln) vs. „Begründungszusammenhang“ (Denken). 
Tab. 1: Erkennen vs. Erklären (Riedl, 2000, S. 53-55).

\begin{tabular}{|c|c|}
\hline Erkennen & Erklären/Verstehen \\
\hline 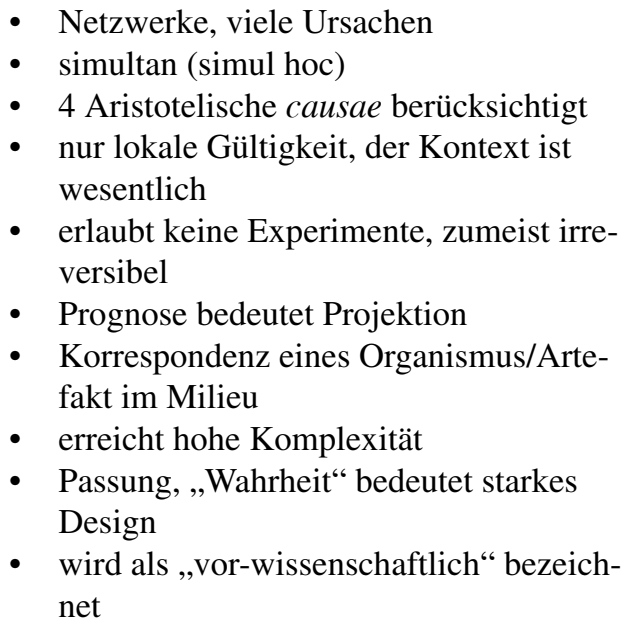 & $\begin{array}{l}\text { - lineare Ursache-Wirkungs-Beziehungen } \\
\text { - } \text { sequentiell (propter hoc) } \\
\text { - } \text { nur Wirkursachen berücksichtigt } \\
\text { globale Gültigkeit beansprucht, Kontext } \\
\text { ausgeblendet } \\
\text { - beruht auf Experimenten, zumeist rever- } \\
\text { sibel } \\
\text { - Prognose bedeutet Vorhersage } \\
\text { - Kohärenz von Elementen in einem Sys- } \\
\text { - tem } \\
\text { - } \text { reduziert Komplexität } \\
\text { - ziehungen } \\
\text { wird als ,wissenschaftlich“ bezeichnet }\end{array}$ \\
\hline
\end{tabular}

Während der ratiomorphe Prozess des Erkennens ein großes Potenzial für den Umgang mit komplexen, evolvierenden Phänomenen besitzt, ist er nicht immer hilfreich für kausale Erklärungen. Entsprechendes gilt umgekehrt. Aber dieses „Dilemma“ ist kein inhärentes Merkmal der Natur der Wissensproduktion, sondern vielmehr eine Konsequenz des dualistischen Konzepts, welches wir dieser aufgezwungen haben. Der Pfad vom Erkennen zum Erklären ist kontinuierlich und zirkulär, manchmal führt er in Sackgassen. Unsere Sprache ist zu arm oder zu stark in der „schwarz-weiß“ Tradition wissenschaftlich geprägten Denkens gefangen, um die wunderbaren Übergänge und Schattierungen von „Grau“ zwischen den Polen auszudrücken.

Das Argument der naturalisierten Erkenntnistheorie erscheint in vielfältigen Formen und Formulierungen. Ein weiterer prominenter Vertreter ist John Dewey (1986). In seiner Sicht bilden intentional getriebene Prozesse des zirkulären Handelns den wesentlichen Kern der Wissenserzeugung. Die Trennung von Denken als reiner Kontemplation und Handeln als der körperlichen Intervention in die Welt wird damit obsolet; es ist gerade umgekehrt: Das Denken ist angewiesen auf zu meisternde Situationen der wirklichen Welt. Es wird angestoßen durch die Notwendigkeit, angemessene Mittel im Hinblick auf erwünschte Konsequenzen auszuwählen. Die projizierte aktive Verbesserung einer unbefriedigenden, problematischen Situation bildet die primäre Motivation für Denken, Entwerfen, und schließlich - in einer verfeinerten, gereinigten, quantitativeren Weise - für wissenschaftliche Forschung und Wissensproduktion. Wissen ist eine Weise des Handelns und „Wahrheit“ wird bei Dewey durch das Konzept der ,gerechtfertigten Behauptbarkeit“" (warranted assertibility) ersetzt. 
Schöns (1983) Erkenntnistheorie der „reflective practice“ kann als designbezogene Umsetzung dieser Konzepte angesehen werden. Es ist gerade dieses spezifische/unspezifische Muster, welches Cross (2001) als ,designerly ways of knowing“ bezeichnet:

„The underlying axiom of this discipline is that there are forms of knowledge special to the awareness and ability of a designer, independent of the different professional domains of design practice."

\section{Eine weitere Grundlage: Die Theorie sozialer Systeme}

Luhmanns Theorie sozialer Systeme stellt die erforderliche Komplexität bereit, um das schöne, aber zu einfache Konzept des ,ganzen Menschen“ in getrennte Subsysteme aufzuteilen.

Design (oder Entwerfen) ist die Disziplin, welche mit Hilfe von Artefakten kontingente Passungen/Interfaces zwischen Körpern, Bewusstseinen und Kommunikationen herstellt (Frage 2).

Der Wechsel vom Denken in Identitäten zum Denken in Differenzen erlaubt es, mit der transitorischen Natur vermeintlich fixer Konzepte umzugehen.

Wenn wir diese hybride, unordentliche Situation erstmal akzeptiert oder zumindest zur Kenntnis genommen haben, dann können wir fortfahren, Design in der Gesellschaft zu beschreiben. Entsprechend dem von Alexander (1964) und Simon (1969) eingeführten Konzept des Interface agiert Design in der Region zwischen dem Artefaktischen und dem Kontextuellen und ist verantwortlich für die Passung zwischen den Objekten und den Kontexten, in denen sie funktionieren (oder in schlichten Darwinistischen Begriffen: überleben) müssen. Um dies produktiver zu machen, müssen wir die common-sense Begrifflichkeit überwinden und explizit kohärente systemische und evolutionäre Konzepte einführen. Luhmann (1997) verschiebt die Betonung im Systemdenken von der ontologischen Unterscheidung Ganzes/Teil hin zur differenztheoretischen Unterscheidung System/Umwelt, was notwendigerweise auf Beobachtung (= Unterscheidung + Bezeichnung) beruht.

Luhmann unterscheidet (heteronome) mechanische Systeme/Artefakte und (autonome) selbstorganisierende Systeme. Die letzteren umfassen Organismen, Bewusstseine und Kommunikationen als autopoietische Systeme. Organismen agieren im Medium des Lebens, Bewusstseine und Kommunikationen im Medium des Sinns. Wir müssen die operationale Geschlossenheit autopoietischer Systeme respektieren und jegliche zeitliche Entwicklung als Ko-Evolution isolierter Systeme betrachten. Dies vermeidet die Illusion der designerischen Kontrolle sozialer Situationen, die stets Bezug zu allen drei Domänen haben oder, wie John Chris Jones (1970) sagen würde, ,to the whole of life“. 
Tab. 2: Der Wechsel von der Identität zur Differenz (nach Luhmann, 1997).

\begin{tabular}{|c|c|}
\hline Identität & Differenz \\
\hline $\begin{array}{l}\text { Ein privilegierter Beobachter postuliert } \\
\text { einen „Ursprung“. }\end{array}$ & $\begin{array}{l}\text { Die Ursprungsanforderung lautet: „Triff eine } \\
\text { Unterscheidung“. }\end{array}$ \\
\hline $\begin{array}{l}\text { Existenz/Nicht-Existenz als feste Ausgangs- } \\
\text { unterscheidung (Sein/Nichtsein). }\end{array}$ & $\begin{array}{l}\text { Diese unterliegt keiner einschränkenden } \\
\text { Vorschrift. }\end{array}$ \\
\hline $\begin{array}{l}\text { Unterteilung des existierenden Ganzen in } \\
\text { Teile. }\end{array}$ & $\begin{array}{l}\text { Schaffen von marked/unmarked state durch } \\
\text { eine Beobachtung (= Unterscheidung }+ \\
\text { Bezeichnung). }\end{array}$ \\
\hline System/Elemente & System/Umwelt \\
\hline $\begin{array}{l}\text { Es gelten die logischen Gesetze } \\
\text { der Identität, } \\
\text { der Widerspruchsfreiheit, } \\
\text { des ausgeschlossenen Dritten. }\end{array}$ & $\begin{array}{l}\text { Die Einheit der Differenz von marked und } \\
\text { unmarked state wird die „Form der Unter- } \\
\text { scheidung“ genannt. }\end{array}$ \\
\hline $\begin{array}{l}\text { Es folgt eine strikt zweiwertige Logik: } \\
\text { Aussagen sind wahr/falsch. }\end{array}$ & $\begin{array}{l}\text { Das Wechseln der Seite der Unterscheidung } \\
\text { erlaubt die Negation und den Austausch der } \\
\text { Gegenbegriffe. }\end{array}$ \\
\hline & $\begin{array}{l}\text { Damit wird eine Art von dreiwertiger Logik } \\
\text { eingeführt - tertium datur. }\end{array}$ \\
\hline
\end{tabular}

Die Konsequenzen dieses Ansatzes sollten nicht als normativ oder gar ,anti-humanistisch“ angesehen werden, sondern als methodologisch: Keine Analyse des Bewusstseins wird je etwas über Kommunikation herausfinden und umgekehrt, ebenso wie eine Analyse von Gedankenprozessen niemals etwas über Gehirnprozesse verraten wird, die in der Domäne der lebenden Systeme ablaufen. Bewusstseine können nicht kommunizieren, lediglich Kommunikation kann kommunizieren. Bewusstseine und Kommunikation interagieren mittels Sprache. Sie haben eine Beziehung der „Interpenetration“, d.h., sie machen Gebrauch voneinander, können sich aber nicht gegenseitig kontrollieren. Unsere Körper befinden sich außerhalb unserer Bewusstseine und außerhalb von Kommunikation. In der Theorie ist kein Platz (und kein Bedarf) für das Individuum (,es gibt einfach zu viele davon“). Damit wird die einfache und diffuse Idee „des Menschen“ vermieden, denn es existiert kein Obersystem, welches lebende, mentale und soziale Systeme vereint. Was „der Mensch“ ist, das hängt davon ab, wer wie beobachtet.

Diese Gemengelage von Interaktionen zwischen Bewusstseinen, Kommunikationen, Organismen und Artefakten bildet den evolutionären „Sumpf“, der hier als provisorische Metapher dient. Design geht mit dieser Situation um und hält an der optimistischen Überzeugung fest, dass Prognose im Hinblick auf den Erfolg von Designeingriffen möglich 
sei. Und genau dies, das Unwissen des Designs über sein Unwissen, macht es attraktiv für andere Disziplinen und sogar für die Wissenschaft (Baecker, 2000):

„Man wird das Design als Praxis des Nichtwissens auf unterschiedlichste Interfaces hin lesen können, aber dominierend sind wahrscheinlich die Schnittstellen zwischen Technik, Körper, Psyche und Kommunikation: Wenn man diese „Welten“, die jeweils von einem mehr oder weniger elaborierten Wissen beschrieben werden, miteinander in Differenz setzt, verschwindet dieses Wissen und macht Experimenten Platz, die die Experimente des Design sind. [...] Hier nichts mehr für selbstverständlich zu halten, sondern Auflösungs- und Rekombinationspotential allerorten zu sehen, wird zum Spielraum eines Designs, das schließlich bis in die Pädagogik, die Therapie und die Medizin reicht. [...]“

Dies ist laut Baecker das Skandalon der gespaltenen Kausalität, welches die Systemtheorie sichtbar macht und mit dem Design stets umgegangen ist. Es gibt eine geschlossene Kausalität, d.h., das System kann als kausal beschrieben werden (Wissen), und es gibt eine unbestimmte Kausalität, die impliziert, dass es in der Umgebung eines Systems weitere Wirkursachen gibt, deren Effekte innerhalb des Systems nicht bestimmbar sind, gerade weil die Existenz des System auf seiner Differenz zur Umwelt beruht und auf seiner Fähigkeit Kausalketten zu zerschneiden (Nichtwissen). Genau diese Lücken sind es, welche die Orte für Modelle von Kreativität wie für Versagen bereitstellen. Wir können zwar noch von Kausalität sprechen, sollten aber bedenken, dass es sich dabei nur noch um die Wahl eines Beobachters handelt.

Dies bedeutet einen Wechsel von der Sicht des Designs als einem kausalen Feld mit (noch) einigen weißen Flecken hin zu einem - prinzipiell unvorhersagbaren, nicht-kausalen - Feld mit einigen unverbundenen Inseln von Kausalität, welche sich zumeist auf isolierte, technische oder wissenschaftliche Fakten beziehen. Sie impliziert die Zurückweisung des Konzepts einer wissenschaftlichen Wissensbasis zugunsten eines funktionalen Schemas des Umgangs mit Wissen. Eine Wissensbasis müsste, wegen der notwendigerweise transdisziplinären Natur von Designhandeln, „Alles“ umfassen, wie Friedmans kanonisch anmutende Listen eindrucksvoll demonstrieren, ohne damit jedoch die Wissensinseln zu verbinden. Schließlich bedeutet es die Zurückweisung des Konzepts von Fortschritt. Während Design Passungen zwischen dynamischen Systemen herstellt, die je für sich selbst durchaus Fortschritt verzeichnen können, gibt es kein sinnvolles Kriterium für Fortschritt im Design selbst. Design evolviert.

Um noch einen Schritt weiter zu gehen: Design agiert als ein manchmal nützlicher, manchmal nervender Parasit (Serres, 1987), der Interfaces, Kupplungen, Hilfestellungen, Prothesen und Sinn generiert. Design beobachtet permanent das Feld im Hinblick auf Wünsche, unbefriedigte Bedürfnisse, potentielle Verbindungen, alle Gelegenheiten zur Einmischung wahrnehmend, die sich zeigen. Designerische Beobachtung ist stets Beobachtung zweiter Ordnung (Beobachtung von Beobachtungen). Kausalität wird, sobald sie von einem Beobachter eingeführt wurde, infolge von unkontrollierbaren Abweichungen und Interaktionen abgebaut. Wem der „Parasit“ zu negativ erscheint, nehme das Bild des Jokers. 


\section{6 Eine dritte Grundlage: Die Theorie der
sozio-kulturellen Evolution}

Die Theorie der sozio-kulturellen Evolution bietet einen brauchbaren Rahmen zur Beschreibung der Nicht-Vorhersagbarkeit von Design-Entwicklungen und Projektergebnissen. Sie beschreibt auf wissenschaftliche Weise die Grenzen kausaler Erklärungsmuster.

Die Ordung und das Chaos des natürlichen und sozialen Lebens wird von einfachen Feedback-Mechanismen hervor gebracht. Die Einsichten über Evolution haben die Evolutionstheorie dazu geführt, sich selbst als ein Ergebnis von Evolution zu betrachten, was unmittelbar zur evolutionären Erkenntnistheorie führt. Es gibt zahlreiche Evidenzen für implizite, evolutionäre Konzepte in Designtheorien und -methoden, selbst in den vermeintlich „rigidesten“ Ansätzen (siehe etwa Roozenburg \& Eekels, 1991, fig. 5.12). Es gibt sogar explizite, evolutionäre Konzepte. Hybs \& Gero (1992) beschreiben Artefakte als Entitäten, welche in der feindlichen Umgebung des Marktes um ihr Überleben kämpfen. Um zu originelleren Beschreibungen zu gelangen, sollte das verallgemeinerte Konzept von Evolution noch rigoroser auf Design angewendet werden. Das Folgende bezieht sich wieder auf Luhmann, dessen Theorien engen Bezug zur evolutionären Erkenntnistheorie aufweisen. In seinem Hauptwerk (1997) hat er begonnen, das Konzept der sozialen Evolution auszuarbeiten.

Zunächst basiert Evolutionstheorie auf dem Konzept der Unterscheidung von System/Umwelt. Gerade diese Differenz ermöglicht Evolution. Dann unterscheidet sie nicht historische Epochen, sondern Variation, Selektion und Re-Stabilisierung. Re-Stabilisierung ist die wesentliche Bedingung dafür, dass Variation und Selektion überhaupt stattfinden können. Evolutionstheorie dient der Entfaltung des Paradoxes von der Wahrscheinlichkeit des Unwahrscheinlichen und erklärt auf diese Weise die Entstehung von Formen und Substanzen aus dem Zufälligen. Indem die Theorie das terminologische Rahmenwerk der Weltbeschreibung vom Kopf auf die Füße stellt, entlastet sie die Ordnung der Dinge von jeglicher Fessel an einen Ursprung oder Form gebenden Anfang. Evolutionstheorie handelt weder von Fortschritt, noch liefert sie Projektionen oder Interpretationen der Zukunft. Das Konzept der autopoietischen Systeme erzwingt darüber hinaus eine Revision der Theorie der „Anpassung“, welche nun Bedingung und nicht etwa Ziel oder Ergebnis von Evolution ist. Solange die Autopoiesis fortdauert, ist es möglich, auf der Grundlage von Anpassung immer riskantere Spielarten von Nicht-Anpassung zu produzieren.

Die drei getrennten Prozessschritte der Evolution können den Komponenten der Gesellschaft, betrachtet als kommunikatives System, zugeordnet werden:

- Variation variiert die Elemente des Systems, d.h. Kommunikationen. Variation bedeutet hier abweichende, unerwartete, überraschende Kommunikation. Dies kann einfach das Infragestellen oder Zurückweisen von Sinnerwartungen bedeuten. Variation produziert Rohmaterial und ermöglicht weitere kommunikative Anschlussmöglichkeiten und damit Sinnpotentiale. 
- Selektion bezieht sich auf die Strukturen des Systems. Strukturen bestimmen die Kreation und den Gebrauch von Erwartungen, welche wiederum Kommunikationsprozesse bestimmen. Positive Selektion bedeutet die Auswahl sinnvoller Beziehungen, die einen Beitrag zum Aufbau oder zur Stabilisierung von Strukturen versprechen. Selektionen haben die Funktion von Filtern, welche die Ausbreitung von Variationen kontrollieren. Religion war ein derartiger Filter. Die symbolisch generalisierten Medien Wahrheit, Geld, Macht fungieren als Filter in modernen Gesellschaften.

- Re-Stabilisierung bezieht sich auf den Zustand eines evolvierenden Systems nach einer positiven/negativen Selektion. Sie ist zuständig für die System-Kompatibilität der Selektion. Selbst negative Selektionen erfordern Re-Stabilisierung, weil sie im Systemgedächtnis verbleiben. Heute wird die Stabilität selbst ein mehr und mehr dynamisches Konzept, welches indirekt als Anreger für Variation agiert.

Variation, Selektion und Re-Stabilisierung können durchaus auf die empirische Realität evolvierender Systeme bezogen werden und erlauben damit die Neu-Interpretation historischer Wirklichkeiten im Licht der Evolutionstheorie. Zum Beispiel:

- Frühe segmentierte Gesellschaften (Familien, Clans, ...), in denen Kommunikation meist durch Interaktion zwischen Anwesenden passiert, benötigt kaum die Unterscheidung von Variation und Selektion, weil jede Interaktion auf unmittelbare Zustimmung oder Ablehnung zielt.

- Stratifizierte, hierarchische Gesellschaften haben Probleme mit der Unterscheidung von Selektion und Re-Stabilisierung, weil Stabilität das Ausschlag gebende Kriterium für Selektion darstellt.

- Moderne, ausdifferenzierte Gesellschaften unterscheiden Variation und Selektion sowie Selektion und Re-Stabilisierung, haben aber Probleme mit der Unterscheidung von ReStabilisierung und Variation, weil Stabilität heute einen extrem dynamischen Charakter angenommen hat und den Antrieb für evolutionäre Variation („Innovation“) darstellt. Hier können wir Design, die Erzeugung von Varietät, als konstitutives Element von Modernität identifizieren.

Zurück zum Design: Die Gegenwart markiert keinesfalls die Wellenfront des Fortschritts, sondern besteht aus dem, was aus der Vergangenheit übrig geblieben ist. Und so leben wir nicht in der besten aller möglichen Welten. Harmonie, wenn sie überhaupt besteht, ist ,poststabiliert" und wird in unseren Erzählungen kreiert. Das Studium fehlgeschlagener Innovationen (,Floppologie“) könnte ein vielversprechender Ansatz zur Verbesserung von Designqualität sein. Die „dunkle Seite“ des Feldes ist vermutlich reicher als das Reservoir der „,best practice“. 


\section{7 Mind the gaps! - Kontrolle und Vorhersage
sind beschränkt}

Es gibt zwei grundsätzliche Probleme im Zusammenhang mit systemischen Wissenslücken:

(1) Die Lücken zwischen den im Design involvierten autopoietischen Systemen: Der fundamentale systemische „Eigensinn“ wird mittels der hübschen und gebräuchlichen Begriffe „Kreativität“, „Subjektivität“, „Werte“, „Trends“, ... bezeichnet oder eher verschleiert.

(2) Die Lücken zwischen den evolutionären Mechanismen, die im Design involviert sind: Variation, Selektion und Re-Stabilisierung sind notwendigerweise kausal entkoppelt, um die „kreative“ Erzeugung des Neuen zu ermöglichen.

Das bis hierher Gesagte erlaubt die folgende Zusammenfassung: Entwerfen besteht aus dem Umgang mit interagierenden/ko-evolvierenden autopoietischen Systemen (Organismen, Bewusstseine und Kommunikationen) und Artefakten. Zufällige Mutationen ebenso wie bewusste Entscheidungen und Verbindungen im kommunikativen Netz initiieren offene Prozesse der Selbstorganisation. Positive und negative Feedback-Mechanismen interagieren und produzieren wechselnde Muster, welche zu Zeiten relativ stabile Formen annehmen können, die wir dann zum Beispiel Moden oder Trends oder Stile nennen. Diese Art der wechselseitigen Kausalbeziehungen impliziert die Unmöglichkeit gerichteter Kontrolle über irgendeinen Satz von Variablen. Eingriffe werden mit hoher Wahrscheinlichkeit Rückwirkungen im Gesamtsystem haben. Auch wenn es manchmal möglich ist, einen ersten „Kick“ zu geben, der das System in eine gewünschte Richtung bewegt, müssen wir uns immer bewusst sein, dass diese Anstöße nicht wirklich die Ursache der Endergebnisse sind. Sie stoßen lediglich Transformationen an, die in der Logik der beteiligten Systeme eingebettet sind. Wir können zwei Problemfelder identifizieren: (1) Kontrolle, aufgrund der System/Umwelt Unterscheidung, und (2) Vorhersage, aufgrund der Unterscheidung von Variation/Selektion/Re-Stabilisierung.

\section{(1) Das Problem der Kontrolle:}

Die Luhmannsche Systemtheorie stellt eine Landkarte der möglichen Lücken im Hinblick auf diese Interventionen, die wir Designaktivitäten nennen, bereit. Es gibt folgende Lücken produzierende Kombinationen:

- Organismen/Artefakte

- Bewusstseine/Artefakte

- Kommunikationen/Artefakte

- Organismen/Kommunikationen/Artefakte

- Bewusstseine/Kommunikationen/Artefakte

- Organismen/Bewusstseine/Artefakte und

- Organismen/Bewusstseine/Kommunikationen/Artefakte. 
Wir nehmen an, dass Artefakte als Artefakte funktionieren; hier liegt nicht die Hauptaufgabe des Designs. Ausgehend von den autopoietischen Systemen lassen sich die Lücken, welche stets in Wechselbeziehung und (je nach Designaufgabe) unterschiedlichen Anteilen auftreten, illustrieren:

- Organismen $\rightarrow$ die „Funktionslücke“, die anzeigt, dass es keine triviale Aufgabe ist, ein Artefakt an einen Körper anzupassen. Körper können nicht sprechen, etwa beim Entwurf eines rückenfreundlichen Bürostuhls ...

- Bewusstseine $\rightarrow$ die „Geschmackslücke“, die anzeigt, dass es keine triviale Aufgabe ist, individuelle Bewusstseine zu koordinieren, etwa zur Optimierung einer Lösung für eine bestimmte Zielgruppe auf dem deutschen Markt. Sie sind alle verschieden und können nicht in klarer und bestimmter Weise ihren Geschmack kommunizieren ...

- Kommunikationen $\rightarrow$ die „Modelücke“, die anzeigt, dass es keine triviale Aufgabe ist, die Vielfalt von aus individuellen Bewusstseinen per Kommunikation gewonnenen Informationen in die Gestalt eines Artefakts zu transformieren, etwa zur Planung einer neuen Kollektion von Haushaltsartikeln für den türkischen Markt ...

\section{(2) Das Problem der Vorhersage:}

Die drei getrennten und unabhängigen Komponenten des Evolutionsprozesses erzeugen weitere Kausalitäts-Spaltungen:

- Variation zielt auf Alternativen. Dies scheint unproblematisch, weil Designer-Bewusstseine reichlich „Kreativität“ bereitstellen, was zunächst ausreicht, um die Vielfalt der Auswahl zu erhöhen. Dies ist die ,zeitlose“ Aufgabe des Entwerfens ...

- Selektion zielt auf die Passung der erzeugten Alternativen in Strukturen. Dies ist tatsächlich ein Problem, denn es sind zwar kommunikative Strukturen erkennbar, nicht aber ihre zukünftige Stabilität. Wenigstens bis zu einem bestimmten Grade ist Designforschung in der Lage, existierende Strukturen zu untersuchen ...

Einzelaspekte können durch isolierte Ansätze behandelt werden: Organismus-ArtefaktLücken mit Hilfe von Ergonomie, Bewusstseins-Artefakt-Lücken mit Hilfe der kognitiven Ergonomie, Kommunikations-Artefakt-Lücken mit Hilfe von Marktforschung, etc.

- Re-Stabilisierung zielt auf die Integration der ausgewählten Alternativen. Hier gibt es kaum Vorhersagbarkeit, denn es handelt sich um eine Frage der langfristigen Lebensfähigkeit neuer Komponenten in kommunikativen Systemen. Futures Studies und Szenarioplanung befassen sich mit evolvierenden Systemen dieser Art...

Designaktivitäten intervenieren in das Beziehungsgeflecht ko-evolvierender autopoietischer Systeme. Dies geschieht mittels Artefakten, welche vorgeben, diese Beziehungen zu verbessern. Das grundsätzliche Problem besteht weder in fehlender individueller Kreativität noch in mangelhafter Planung, sondern im unkontrollierbaren und nicht vorhersagbaren Verhalten von Körpern, Bewusstseinen und Kommunikationen in der Umgebung der Artefakte. Designaktivitäten sind an die Zeitstrukturen anderer Systeme wie Wirtschaft, Wissenschaft, Politik angekoppelt. Design hat keine „Eigenzeit“. Design evolviert „dazwischen“. Das am weitesten entwickelte, fast universelle Instrument zur Überbrückung derartiger Lücken stellt die Sprache dar, welche Kommunikation ermöglicht. Funktionierende Kommunikation ist bekanntlich unwahrscheinlich. Funktionierendes Design ist noch unwahrscheinlicher... 


\section{8 Veränderungen in Gesellschaft
und Wissensproduktion}

Wissenschaft sieht sich, gerade wegen ihres Erfolgs, einer Veränderung ausgesetzt, einem Wechsel hin zu projektorientierten Formen wissenschaftlicher Praxis. Die Unfähigkeit, infolge der nun auch hier auftretenden Wissenslücken, mit den Folgen umzugehen, führt zu einer „Krisis des Wissens“: es gibt kein reines „theoretisches“ Wissen mehr, sondern nur praktisches Wissen über den Umgang mit Theorie.

Eine „Sozialisierung der Wissenschaft" findet statt: Wissenschaft wird sichtbarer, wird beobachtet und gerät unter wachsende öffentliche „Kontrolle“.

Der bisher entwickelte Gedankengang wird durch Veränderungen in der Gesellschaft und in der Wissensproduktion unterstützt. Die entwickelte Moderne glaubte an Planung, Vorhersagbarkeit, Fortschritt und an die zwangsläufige „Verwissenschaftlichung der Gesellschaft“. Das dritte Viertel des 20. Jahrhunderts erlebte den Höhepunkt der wissenschaftsbasierten Professionalisierung und erkannte zugleich deren Defizite (z.B. Schön, 1983). Seit den 1970ern zeigen sich massive Veränderungen in den Mustern der Wissensproduktion und in der Gesellschaft selbst, die, in positiver Begrifflichkeit, als „Wissensgesellschaft“ (Bell, 1973), oder, negativer und „,deutscher“, als „Risikogesellschaft“ (Beck, 1986) bezeichnet wurden. Um das unangemessene Konzept der Postmoderne zu vermeiden, können wir feststellen, dass die Moderne reflexiv wird. Design ist diesen Entwicklungen gegenüber höchst sensibel gewesen, wie etwa der radikale Wechsel im Design Methods Movement um 1970 zeigt.

Unsere Aktivitäten werden projektiver und machtvoller, und gleichzeitig erkennen wir die Unfähigkeit, ihre Konsequenzen vorherzusagen. Wir müssen solides, wissenschaftliches und technologisches Wissen über Interventionen mit reiner Ignoranz (Nichtwissen) bezogen auf ihre psychischen, sozialen, ökonomischen und ökologischen Folgen zu ganz neuen Handlungsmodellen verknüpfen. Kurzfristige Determiniertheit verwandelt sich in langfristige Ungewissheit. Die „Krisis des Wissens“ (Willke, 2002) offenbart unsere Unfähigkeit im Umgang mit Nichtwissen. Frühere Gesellschaften hatten dafür angemessene Mechanismen: traditionelle Gesellschaften etwa in der Funktion der Religion oder industrielle Nationalstaaten in der auf Macht begründeten Funktion des freien Unternehmertums. Wenn wir auf das Konzept der Form zurückgreifen (zur Illustration der beobachtungsleitenden Unterscheidungen, siehe Kap. 5), dann erkennen wir, dass die entsprechenden Formen des Wissens in diesen Fällen Wissen/Glauben und Wissen/Macht sind. Beide Formen prozessieren ihre Entscheidungen nicht im Feld des Wissens, sondern auf dessen Gegenseite. In hochkomplexen, säkularen Gesellschaften müssen wir der Grausamkeit eines Wissenskonzepts ins Gesicht sehen, das seine andere Seite weder im Glauben noch im Machen findet, sondern im Nichtwissen selbst. Wir sind nicht länger in der Lage, die Last des Nichtwissens in die Ferne transzendentaler Symbolisierungen oder in die Unmittelbarkeit des forcierten Machens zu verschieben.

Diese Konzeptverschiebung mag als akademische Übung erscheinen, aber sie verdeutlicht die Möglichkeit und Notwendigkeit, kognitiven Sackgassen zu entkommen, indem wir die Probleme von der anderen Seite der Unterscheidung her angehen. Pragmatisch gesprochen: die 
Aneignung von Kompetenz im Umgang mit Ungewissheit bedeutet beispielsweise, dass wir die richtigen Fehler schneller als andere machen müssen.

Nowotny et al. (2001) charakterisieren diese Entwicklung als eine Verschiebung von einer „Mode-1-“ zu einer „Mode-2“-Gesellschaft. Die Schnittstellen zwischen Staat, Märkten und Kultur werden zunehmend verwischt. Die relativ autonomen Räume, welche diese Systeme besetzt hatten, waren Ergebnisse der modernen Differenzierung, ebenso wie die Wissenschaft. In scheinbarem Gegensatz zu Luhmann wird die These vertreten, dass das Schema der funktionalen Differenzierung sich in Teilen auflöst. Das neue Programm des französischen CNRS ${ }^{1}$ belegt diese Verschiebung von traditionellen Disziplinen hin zu interdisziplinären Problemfeldern. Darüber hinaus führt das CNRS die Institution von „Bürgerkonferenzen“ ein (Frankfurter Allgemeine Zeitung, 26.03.2002). In der Mode-2-Gesellschaft zeigt sich eine Entwicklung, die man als „Sozialisierung von Wissenschaft“ bezeichnen könnte oder als Wechsel von der Mode-1- zur Mode-2-Wissensproduktion. Wissenschaft und Gesellschaft werden transgressiv, d.h., dass nicht nur die Wissenschaft zur Gesellschaft spricht (was sie immer getan hat), sondern, dass die Gesellschaft nun ihrerseits die Wissenschaft anspricht. Innovation bildet das Kernstück eines neuen Kontrakts zwischen Wissenschaft und Gesellschaft. Wissenschaft begibt sich in die Agora (Nowotny et. al., 2001, S. 201): , Science is no longer outside, either as a cognitive or quasi-religious authority or as an autonomous entity with its special access to the reality of nature. "

Gerade wegen ihrer Erfolge gerät die Wissenschaft unter den Druck, effektive Lösungen für eine weite Spanne von zunehmend komplexen Problemen zu liefern. Auf diese Weise wird die Wissenschaft zur Produktion von kontextualisiertem Wissen gedrängt. Kontextualisierung geschieht durch die Verschiebung von einem „Segregations-“ zu einem „Integrationsmodell“, vom disziplinären zum Problemfokus, oder: von der gegenstandsbezogenen Wissenschaft zur projektorientierten Forschung. Dies ist gekennzeichnet durch höhere Ungewissheit infolge der „darwinistischen“ Mechanismen von Variation und selektiver Re-Stabilisierung sowie durch die größere Aufmerksamkeit für den Platz und die Rolle von „Menschen“ dabei: aktiv in die Wissensproduktion involviert entweder als Gegenstände der Untersuchung oder als Adressaten zu entwickelnder Politiken. Mode-2-Wissensproduktion impliziert, dass:

- die Trennung von Grundlagen- und angewandter Forschung sich verwischt (Beispiel Quantencomputer),

- die Trennung von natürlich und künstlich, von Wissenschaft (was ist) und Design (was sein könnte) unscharf wird (Beispiel genetisches Design),

- die Unterscheidung von Fakten und Werten ein Problem wird,

- der Kontext der Anwendung sich ausweitet zu einem Kontext der Auswirkungen,

- der Fokus von verlässlichem zu sozial robustem Wissen wechselt,

- das Konzept des „Entdeckungszusammenhangs“ im Gegensatz zum „Begründungszusammenhang“" obsolet wird,

- $\quad .$.

Aus dieser Perspektive betrachtet, gibt es kein „theoretisches“ Wissen mehr, sondern nur praktisches Wissen über den Umgang mit Theorie. Es gibt kein „,abstraktes“Wissen, sondern lediglich praktisches Wissen im Umgang mit Abstraktionen.

1. Centre national de la recherche scientifique (vergleichbar mit der deutschen Max-Planck-Gesellschaft). 
Der „harte“ epistemische Kern der autonomen Wissenschaft, um dessen Artikulation und Etablierung die Wissenschaftler Jahrhunderte lang gekämpft haben, wird ,weicher“. Der Ort ist nicht leer, sondern bevölkert und heterogen. Es handelt sich nicht um einen plötzlichen Paradigmenwechsel von Wissenschaft zu Nicht-Wissenschaft oder von universellen, objektiven Standards zu einem lokal bestimmten Relativismus, sondern um die vorerst letzte Stufe der Angleichung an eine komplexer werdende Realität. Möglicherweise kann man die Situation als eine Entkopplung der Modernisierung von der Modernität charakterisieren. Die Prozesse der Innovation werden von den Werten, auf denen sie einst paradigmatisch beruhten, getrennt.

\section{9 Design als nicht-moderne Disziplin -
Wissenschaft nähert sich dem Design}

In kleinerem Rahmen war Design immer schon die Expertendisziplin des Umgangs mit Nichtwissen. Design und Wissenschaft basieren auf zirkulären Untersuchungsprozessen. Sie unterscheiden sich (noch) in ihren Zielsetzungen und Ergebnissen und in den Kriterien zur Bewertung dieser Ergebnisse. Dennoch: Wissenschaft wird zunehmend „design-ähnlich“.

Design ist daher aufgefordert, sein in den Grundzügen nicht-modernes Profil zu schärfen, statt nach der Anpassung an ein sich in Richtung Design veränderndes Wissenschaftskonzept zu streben ( $\rightarrow$ Fragen 2, 4).

Ursprünglich sind die Menschen „universelle Dilettanten“. Die Strukturierung und funktionale Differenzierung von Gesellschaften hat diese einzigartige Trans-Kompetenz entwertet. Design, als ein Produkt der Moderne, entsteht als vermittelnde Schnittstelle zwischen dem zunehmend getrennten Machen und dem Benutzen der Artefakte. Design professionalisiert die Kompetenz des „universellen Dilettantismus“; der poietische menschliche Trieb wird längst durch das Angebot der Do-It-Yourself Industrien kompensiert. Die funktionale Differenzierung der Gesellschaften stellt die paradoxe Grundlage des Designs dar. Paradox deshalb, weil Design gleichzeitig als vorlaute „Un-Disziplin“ diese Separierung ablehnt und sich permanent überall einmischt. In diesem Sinne, wegen seiner Orthogonalität zur operationalen Geschlossenheit der autopoietischen Systeme, steht Design auch quer zu den traditionellen Strategien der Modernisierung. Design ist niemals ganz modern gewesen. Und deshalb kann es möglicherweise als Modell für den Umgang mit den Konflikten der Modernität dienen.

Andererseits gibt es deutliche Anzeichen dafür, dass wissenschaftliche Forschungspraxis den designerischen Wegen des Handelns und Reflektierens ähnelt. Ein „,dritter Weg“, eine nuanciertere und soziologisch sensiblere Epistemologie scheint erforderlich, welche die „weichen“ individuellen, sozialen und kulturellen Visionen ebenso wie den „harten“ Bestand wissenschaftlichen Wissens integriert. Ich habe drei Thesen zum Design formuliert (Jonas, 2001), die auf die Wissenschaft ausgedehnt werden können. 


\section{(1) Design muss passen - Wissenschaft ebenso.}

Dies bezieht sich auf das Interface-Konzept des Designs. Die wachsende Kontextualisierung wissenschaftlicher Praxis verschiebt die Betonung von interner Kohärenz der Modelle und Ergebnisse hin zur Passung in Bezug auf die Kontexte (externe Korrespondenz).

\section{(2) Design endet niemals - Wissenschaft auch nicht.}

Dies bezieht sich auf Design als projektive Disziplin, welche versucht, existierende Situationen in bevorzugte zu verwandeln. Wenn das Problem gelöst ist, wird die Lösung zumeist zum Keim eines neuen Problems. Das neue wissenschaftliche Kriterium der sozialen Robustheit (s.o.) erfordert permanentes Feedback mit dem Kontext in der Institution der „Agora“. Kontextualisierte wissenschaftliche Probleme sind, wie Designprobleme, niemals gelöst (Carroll, 1974, S. 38, 39):

„,Jetzt! Jetzt!‘ rief die Königin. ,Schneller! Schneller!‘ Und nun sausten sie so schnell dahin, daß sie beinahe nur noch durch die Luft segelten und den Boden kaum mehr berührten, bis sie plötzlich, als Alice schon der Erschöpfung nahe war, innehielten, und im nächsten Moment saß Alice schwindlig und atemlos am Boden.

Die Königin lehnte sie mit dem Rücken gegen einen Baum und sagte gütig: ,Jetzt darfst du ein wenig rasten.

Voller Überraschung sah sich Alice um., Aber ich glaube fast, wir sind die ganze Zeit unter diesem Baum geblieben! Es ist ja alles wie vorher!

,Selbstverständlich', sagte die Königin; , was dachtest du denn?

,Nun, in unserer Gegend', sagte Alice, noch immer ein wenig atemlos, ,kommt man im allgemeinen woandershin, wenn man so schnell und lange läuft wie wir eben.

,Behäbige Gegend!‘ sagte die Königin. ,Hierzulande mußt du so schnell rennen, wie du kannst, wenn du am gleichen Fleck bleiben willst. Und um woandershin zu kommen, muß man noch mindestens doppelt so schnell laufen!““

\section{(3) Design ist eine besondere Kunst - Wissenschaft auch.}

Design sollte sich seiner vormodernen Relikte nicht schämen. Es gibt „geheimnisvolle“ Aspekte im Entwerfen, wie immer wir sie nennen: Intuition, Kreativität, Einsicht, oder, was ich bevorzugen würde: Zufall. Heisenberg vergleicht mentale Bilder mit den schließlich resultierenden mathematischen Modellen und schlägt sogar in den „,sehr harten“ Wissenschaften eine komplementäre Sicht der Wissensproduktion vor (Miller, 1996, S. 319, 320):

"[...] And, of course, then you try to give this picture some definite form in words or in mathematical formula. Then what frequently happens later on is that the mathematical formulation of the 'picture' or the formulation of the 'picture' in words turns out to be rather wrong. Still the experimental guesses are rather right, that is, the actual 'picture', which you had in mind was much better than the rationalization which you tried to put down in the publication. That is, of course, a quite normal situation, because the rationalization, as everyone knows, is always a later stage and not the first stage. [...]“ 
In einem strikten Mode-1-Sinne ist Design nie modern gewesen und die Disziplin sollte nicht um Modernität kämpfen, zumal in einer Situation, wo auch andere „Wissenschaften vom Künstlichen“ (BJM, 2001) wichtige Aspekte von Modernität hinter sich lassen. Design kann als Agentur der Modernisierung (Innovation) beschrieben werden, entkoppelt von den Idealen der Modernität, angesiedelt zwischen den etablierten wissenschaftlichen und professionellen Sphären und Expertendisziplinen. Und gerade dies ist seine besondere Stärke. Wiener (1948) hat betont, dass die fruchtbarsten Felder für das Gedeihen der Wissenschaft diejenigen sind, die im unbeachteten „Niemandsland“ zwischen den anerkannten Disziplinen liegen. Die Kybernetik etwa war ein Produkt konkreter Entwurfsprobleme. Aus diesen fruchtbaren Niemandsländern können sich weitere Disziplinen entwickeln. Design selbst wird jedoch im Sumpf bleiben, oder genauer: Design wird der evolutionäre Sumpf bleiben, wo sich die potentiellen Sinnpfade bilden und materialisieren; oder das noch unverdrahtete Gehirn, wo die Axonen ihre Verbindungen suchen: hypothetisch, explorativ, spekulativ...

\section{Glauben wir nicht an Grundlagen - Design als ironische Disziplin}

Je „wahrer“ und damit normativer Designgrundlagen zu sein vorgeben, desto kontraproduktiver werden sie. Systemische Ironie ist die optimale Strategie gegen einschläfernde Wahrheiten und fest gefügte Standards. Die temporale Zirkularität (Spiralität) von Machen, Beobachten, Theoretisieren, Planen, Machen, ... mit den genannten Konsequenzen hinsichtlich der Konsequenzen scheint die ultimative Grundlage zu bilden.

In der Designforschung können wir (vorerst) nur Feyerabend folgen (question 5).

Feierabend!

Zusammenfassend: wir stehen vor der paradoxen Situation steigender Manipulationsmacht durch Wissenschaft und Technologie, sogar die natürliche Evolution selbst betreffend, und zugleich abnehmender prognostischer Kontrolle. Design agiert evolutionär, sowohl was seine Funktion betrifft (Entwurfsprozesse) als auch die Schaffung eigener unwahrscheinlicher disziplinärer Ordnung (Strukturen und theoretische Grundlagen) betreffend. Designtheorie muss eine Passung zwischen sich selbst (als Artefakt) und seiner Umgebung herstellen, die aus sozialen Bedingungen, modischen Theorien, Wertvorstellungen, etc. besteht. Es gibt Selbstähnlichkeit zwischen den Prozessen des Entwerfens und den Prozessen der disziplinären Entwicklung. Möglicherweise ist temporale Zirkularität (wie etwa in der Aktionsforschung) das einzig brauchbare Prozessmodell in der Designpraxis wie in der Designtheoriebildung.

Ich schlage eine perspektivische Kehrtwendung vor, oder, etwas bombastischer: einen „Paradigmenwechsel“. Der wichtigste Lerneffekt nach 40 Jahren Designforschung könnte darin bestehen, den Anspruch auf Erklärung und Begründung wie auf Vollständigkeit und Kohärenz der theoretischen Modelle zu reduzieren oder aufzugeben. Funktionale Äquivalente, welche die Prozessdynamik auf den unterschiedlichen Ebenen beschreiben, scheinen mehr zu versprechen als normative Wissensbasen und Regeln. Der Gewinn an Interpretationspotential 
wird den Verlust an Erklärungskraft mehr als ausgleichen. Statt die Inseln scheinbarer wissenschaftlicher Rationalität mit ungeeigneten Mitteln auszudehnen (was sich häufig als nicht tragfähig erweist), sollten wir mutig die Grenze vom Wissen zum Nichtwissen überschreiten. Und auf dieser Seite der Grenze können wir (wissenschaftlich begründet!) die Gebiete sicherer Nicht-Vorhersagbarkeit bestimmen.

Das Akzeptieren der Grenzen wissenschaftlicher Begründungen in der Schaffung des Neuen erfordert tatsächlich ein neues Selbstkonzept. Broadbent (2002) schlägt ein designerisches „Leitsystem“ der soziokulturellen Evolution vor, was reichlich ambitioniert, fast arrogant erscheint. Ich würde vorschlagen, ein wenig bescheidener zu sein. Selbstverständlich sollten wir aber an dem großen, aber sympathischen (und etwas naiven) und professionellen Anspruch festhalten, die „Lebensqualität“ zu befördern. Dies bedeutet, dass unsere Theorie Züge einer ironischen Theorie annehmen muss, denn nur Ironie ist in der Lage, Bescheidenheit mit Arroganz zu kombinieren:

- Wir kennen die Sokratische Ironie („Ich weiß, dass ich nichts weiß.“) als den Kern des maieutischen Ansatzes, der immer schon ein Element guter Planung war.

- Wir kennen die romantische Ironie als das elegante Spiel des Genialen. Auf die eigenen Fähigkeiten angewendet, wird mittels dieser Art von Ironie die eigene Überlegenheit zugleich dekonstruiert und bestätigt.

- Wir kennen Rortys (1989) Ironiekonzept mit seiner tiefen Skepsis in Bezug auf abgeschlossene und endliche Vokabulare.

- Und wir haben die „systemische Ironie“, die beansprucht, eine Kompetenz des reflektierten Umgangs mit fundamentaler Ungewissheit darzustellen. Dies bedeutet, dass Letztere nicht durch geduldige Reflektion aufgelöst werden kann, sondern dass sie sich in der Reflektion sogar ausweitet, weil jede Beobachtung neue Felder (noch) nicht beobachteter Phänomene eröffnet. Die andere Seite der systemischen Ironie ist nicht Gewissheit, sondern nichts anderes als das unvermeidbare Paradox des Wissens. In eben diesem Sinne erklärt Schlegel (zitiert nach Willke, 2002): „Ironie ist die Form der Paradoxie. “ Es ist „klares Bewußtsein ... des unendlich vollen Chaos. “

\section{$11 \quad$ Literatur}

Albert, H. (1968). Traktat über kritische Vernunft. Tübingen.

Alexander, C. (1964). Notes on the Synthesis of Form. Cambridge, Mass.: Harvard University Press.

Baecker, D. (2000). Die Theorieform des Systems. Soziale Systeme 6 (2000), H. 2, S. $213-$ 236. Opladen: Leske + Budrich.

Beck, U. (1986). Risikogesellschaft. Auf dem Weg in eine andere Moderne. Frankfurt a.M.: Suhrkamp.

Bell, D. (1973). The Coming of Post-Industrial Society. London: Heinemann. 
BJM (2001). British Journal of Management Volume 12, Special Issue on Mode-2 knowledge production, December 2001.

Blackmore, S. (1999). The Meme Machine. Oxford: Oxford University Press.

Broadbent, J. (2002). Generations in design methodology. Common Ground. International Conference of the DRS, Brunel University, London 5-8 September 2002.

Buchanan, R. (2001). Children of the Moving Present: The Ecology of Culture and the Search for Causes in Design. Design Issues 17: 1, 67-84.

Campbell, D.T. (1974). Evolutionary epistemology. In Schlipp, P.A. (ed.), The Philosophy of Karl Popper, Vol. 1, 413-463. La Salle, IL: Open Court Publishing.

Carroll, L. (1974). Alice hinter den Spiegeln. Frankfurt a.M.: Insel Verlag.

Common Ground (2002). Proceedings of DRS International Conference, Brunel University, London 5-8 September 2002, edited by David Durling and John P. Shackleton.

Cross, N. (2001). Designerly Ways of Knowing: Design Discipline Versus Design Science. Design Issues 17: 3, 49-55.

Dawkins, R. (1976). The Selfish Gene. Oxford: Oxford University Press.

Dewey, J. (1986). Logic: The Theory of Inquiry Carbondale: Southern Illinois University Press. Original 1938.

Dunne, A. \& Raby, F. (2001). Design Noir: The Secret Life of Electronic Objects. Basel Boston Berlin: August/Birkhäuser.

Feyerabend, P. (1976). Wider den Methodenzwang. Skizze einer anarchistischen Erkenntnistheorie. Frankfurt a.M.: Suhrkamp.

Friedman, K. (2001). Problem and paradox in foundations of design. In W. Jonas (ed.), the basic PARADOX. http://www.thebasicparadox.de.

Glanville, R. (2001). Researching design and designing research. In W. Jonas (ed.), the basic PARADOX. http://www.thebasicparadox.de.

Hybs, I. \& Gero, J. S. (1992). An evolutionary process model of design. Design Studies Vol. 13, No 3, July 1992, 273-290.

Jonas, W. (1999). On the Foundations of a 'Science of the Artificial'. useful and critical - the position of research in design. International Conference, UIAH, Helsinki, September 1999.

- (2000). The paradox endeavour to design a foundation for a groundless field. International conference on design education in the university. Curtin University, Perth, December. 2000.

- (2001-2003). the basic PARADOX. http://www.thebasicparadox.de.

- (2002). Common Ground - a product or a process? Common Ground. International Conference of the DRS, Brunel University, London, 5-8 September 2002. 
Jonas, W. \& Meyer-Veden, J. (2004). Mind the gap! - On knowing and not-knowing in design. Bremen: Hauschildt Verlag.

Jones, J.C. (1970) Design Methods. Seeds of human futures. London: John Wiley \& Sons.

Latour, B. (1998). Wir sind nie modern gewesen - Versuch einer symmetrischen Anthropologie. Frankfurt a.M.: Fischer. Original 1991.

Love, T. (2002). Learning from the design-science paradox: new foundations for a field of design. In W. Jonas (ed.), the basic PARADOX. http://www.thebasicparadox.de.

- (1984). Soziale Systeme. Grundriß einer allgemeinen Theorie. Frankfurt a.M.: Suhrkamp.

Luhmann, N. (1997). Die Gesellschaft der Gesellschaft. Frankfurt a.M.: Suhrkamp.

Miller, A. I. (1996). Insights of Genius. Imagery and Creativity in Science and Art. New York: Copernicus.

Nowotny, H. \& Scott, P. \& Gibbons, M. (2001). Re-Thinking Science. Knowledge and the Public in the Age of Uncertainty. Cambridge, UK: Polity Press.

Riedl, R. (2000). Strukturen der Komplexität. Eine Morphologie des Erkennens und Erklärens. Berlin Heidelberg New York: Springer.

Roozenburg, N.F.M. \& Eekels, J. (1991). Product Design: Fundamentals and Methods Chichester: Wiley.

Rorty, R. (1989). Contingency, irony, and solidarity Cambridge, UK: Cambridge University Press.

Schön, D. A. (1983). The Reflective Practitioner. How Professionals Think in Action. New York: Basic Books.

Serres, M. (1987). Der Parasit. Frankfurt a.M.: Suhrkamp.

Simon, H.A. (1969). The Sciences of the Artificial. Cambridge, Mass.: MIT Press.

Wiener, N. (1948). Cybernetics or control and communication in the animal and the machine. Cambridge, Mass.: MIT Press.

Willke, H. (2002). Dystopia. Studien zur Krisis des Wissens in der modernen Gesellschaft Frankfurt a.M.: Suhrkamp. 